УДК 519.87.53

Корректность математической модели слабосжимаемой вязкоупругой среды Максвелла*

\author{
Е.Н. Журавлева ${ }^{1,2}$ \\ ${ }^{1}$ Институт гидродинамики им. М.А. Лаврентьева СО РАН (Новосибирск, \\ Россия) \\ ${ }^{2}$ Новосибирский государственный университет (Новосибирск, Россия)
}

\title{
Correctness of the Mathematical Model of a Weakly Compressible Viscoelastic Maxwell Medium
}

\author{
E.N. Zhuravleva ${ }^{1,2}$
}

${ }^{1}$ Lavrentyev Institute of Hydrodynamics of the Siberian Branch of the Russian Academy of Sciences (Novosibirsk, Russia)

${ }^{2}$ Novosibirsk State University (Novosibirsk, Russia)

На базе модели сжимаемой вязкоупругой среды Максвелла с постоянной динамической вязкостью и временем релаксации построена математическая модель движения баротропной среды, плотность которой мало отличается от постоянной. Поведение среды описывается в терминах вектора скорости, давления и тензора напряжений. Релаксационное соотношение для сжимаемой среды выписано для всего тензора напряжений без выделения в нем девиатора. Для описания слабосжимаемой среды проведена линеаризация системы уравнений, описывающих двумерное движение сжимаемой вязкоупругой среды Максвелла. Линеаризация проведена на состоянии покоя с постоянной ненулевой плотностью. В результате выделено уравнение для определения давления и симметрическая гиперболическая линейная система для компонент вектора скорости и тензора напряжений. Сохранение свойства гиперболичности и симметрический вид полученной системы позволяют использовать хорошо развитую теорию гиперболических уравнений. В рамках линейного приближения найдена область единственности задачи Коши и выписано условие корректности начально-краевой задачи для математической модели двумерного движения слабосжимаемой вязкоупругой среды Максвелла.

Ключевые слова: сжимаемая вязкоупругая среда Максвелла, баротропная жидкость, линеаризация, гиперболическая система, корректность начально-краевой задачи.

\section{DOI 10.14258/izvasu(2018)1-15}

* Работа выполнена при финансовой поддержке Российского фонда фундаментальных исследований (код проекта 16-01-00127)
A mathematical model of barotropic medium motion with medium density close to a constant is developed on the basis of the model of compressible viscoelastic Maxwell medium with constant dynamic viscosity and relaxation time. Medium behavior is described by the velocity vector, the pressure, and the stress tensor. The relaxation relation for a compressible medium is produced for the entire stress tensor without the selection of a deviator in it. A linearization of the system of equations describing the two-dimensional motion of a compressible viscoelastic Maxwell medium was carried out to describe a weakly compressible medium. The linearization is carried out for the state of rest with a constant nonzero density. It results in the equation for determining the pressure and a symmetric hyperbolic linear system for components of the velocity vector and the stress tensor. Hyperbolicity and symmetric form of the obtained system are preserved, thus, allow using the well-developed theory of hyperbolic equations. Using the linear approximation framework, the uniqueness domain of the Cauchy problem is found, and the correctness condition of the initial boundary value problem for the mathematical model of the two-dimensional motion of a weakly compressible viscoelastic Maxwell medium is obtained.

Key words: a compressible viscoelastic Maxwell medium, barotropic liquid, linearization, a hyperbolic system, correctness of the initial boundary value problem.

1. Введение. Модели поведения вязкоупругой среды Максвелла являются предметом многочисленных математических исследований [1-9]. На основе таких моделей описываются 
поведение металлов под действием импульсных нагрузок, движение расплавов и растворов полимеров. Математическая природа этих моделей зависит от того, в какой мере учитывается сжимаемость среды. Теория сжимаемой вязкоупругой среды Максвелла давно и хорошо развита $[3,4]$. Модели с постоянной плотностью оказались более сложными с математической точки зрения, т.к. они теряют важное свойство гиперболичности. Однако в последнее время появилось много работ, посвященных изучению свойств несжимаемых сред Максвелла: найдены точные решения [5], исследована групповая природа $[6,7]$, изучена корректность математических моделей для различного вида релаксационных соотношений $[8,9]$.

В предлагаемой работе исследуется математическая модель слабосжимаемой баротропной среды Максвелла для плоского случая.

2. Постановка задачи. Математическими характеристиками сжимаемой вязкоупругой среды Максвелла являются: $\rho$ - плотность, $\mu$ - динамическая вязкость и $\tau-$ время релаксации. Две последние величины предполагаются постоянными, а плотность - функцией давления $\rho=$ $\rho(p)$. Считается, что на среду не действуют внешние объемные силы или действуют внешние силы, имеющие потенциал.

Обозначим $\mathbf{V}=(u(x, y, t), v(x, y, t))-$ вектор скорости, $\mathbf{D}$ - тензор скоростей деформации, $\mathbf{P}-$ тензор напряжений

$$
\mathbf{P}=\left(\begin{array}{ll}
\sigma_{1,1}(x, y, t) & \sigma_{1,2}(x, y, t) \\
\sigma_{2,1}(x, y, t) & \sigma_{2,2}(x, y, t)
\end{array}\right) .
$$

Уравнение неразрывности сжимаемой среды имеет вид:

$$
\frac{\partial \rho}{\partial t}+\operatorname{div}(\rho \mathbf{V})=0 .
$$

Уравнение импульса любой сплошной среды, удовлетворяющей принципу напряжений Коши:

$$
\rho\left(\frac{\partial \mathbf{V}}{\partial t}+\mathbf{V} \cdot \nabla \mathbf{V}\right)=\operatorname{div} \mathbf{P} .
$$

Релаксационное соотношение для сжимаемой среды выписывается для всего тензора напряжений P без выделения в нем девиатора [8]:

$$
\tau \frac{\tilde{d} \mathbf{P}}{d t}+\mathbf{P}=2 \mu \mathbf{D}
$$

где $\frac{\tilde{d}}{d t}$ - одна из объективных производных [2]. Для нашего исследования не имеет значения, какая именно производная рассматривается. В дальнейшем для определенности будем использовать вращательную производную Яумана:

$$
\frac{\tilde{d} \mathbf{P}}{d t}=\frac{\partial \mathbf{P}}{\partial t}+\mathbf{V} \cdot \nabla \mathbf{P}-\mathbf{W} \cdot \mathbf{P}+\mathbf{P} \cdot \mathbf{W},
$$

где $\mathbf{W}=\frac{1}{2}\left(\nabla \mathbf{V}-\nabla \mathbf{V}^{T}\right)$.

Заметим, что тензор напряжений является симметричным; для удобства дальнейших выкладок введем следующие обозначения: $\sigma_{1,1}=$ $\alpha, \sigma_{1,2}=\sigma_{2,1}=\beta, \sigma_{2,2}=\gamma$. Таким образом, для шести неизвестных функций $\rho(x, y, t), u(x, y, t)$, $v(x, y, t), \alpha(x, y, t), \beta(x, y, t), \gamma(x, y, t)$ имеем систему шести уравнений:

$$
\begin{array}{r}
\rho_{t}+(\rho u)_{x}+(\rho v)_{y}=0, \\
\rho\left(u_{t}+u u_{x}+v u_{y}\right)-\alpha_{x}-\beta_{y}=0, \\
\rho\left(v_{t}+u v_{x}+v v_{y}\right)-\beta_{x}-\gamma_{y}=0, \\
\tau\left(\alpha_{t}+u \alpha_{x}+v \alpha_{y}\right)-\tau \beta u_{y}+\tau \beta v_{x}- \\
-2 \mu u_{x}+\alpha=0, \\
\tau\left(\beta_{t}+u \beta_{x}+v \beta_{y}\right)+\frac{1}{2}(\tau \alpha-\tau \gamma-2 \mu) u_{y}+ \\
+\frac{1}{2}(\tau \gamma-\tau \alpha-2 \mu) v_{x}+\beta=0, \\
\tau\left(\gamma_{t}+u \gamma_{x}+v \gamma_{y}\right)-\tau \beta v_{x}+\tau \beta u_{y}- \\
-2 \mu v_{y}+\gamma=0 .
\end{array}
$$

Данная система описывает двумерное движение сжимаемой жидкости. Целью нашего исследования является модель слабосжимаемой среды. Для ее получения будем считать, что плотность мало отличается от постоянной и зависит только от давления: $\rho=\rho_{0}+\epsilon \delta p$, где $\rho_{0}-$ некоторая постоянная плотность, $\epsilon$ - безразмерный малый параметр, $\delta$ - величина порядка единицы с размерностью $\left[c^{2} / m^{2}\right]$. Линеризуем систему (1) на состоянии покоя, т.е. на решении

$$
\rho=\rho_{0}, u=v=\alpha=\beta=\gamma=0 .
$$

Приравнивая коэффициенты при малом параметре $\epsilon$, получим линейную систему:

$$
\begin{gathered}
\delta p_{t}+\rho_{0}\left(u_{x}+v_{y}\right)=0, \\
\rho_{0} u_{t}-\alpha_{x}-\beta_{y}=0, \\
\rho_{0} v_{t}-\beta_{x}-\gamma_{y}=0, \\
\tau \alpha_{t}-2 \mu u_{x}+\alpha=0, \\
\tau \beta_{t}-\mu\left(u_{y}+v_{x}\right)+\beta=0, \\
\tau \gamma_{t}-2 \mu v_{y}+\gamma=0 .
\end{gathered}
$$

Заметим, что давление входит только в первое уравнение системы (2a), поэтому будем исследовать отдельно уравнения (2b)-(2f) для определения функций $u(x, y, t), v(x, y, t), \alpha(x, y, t)$, $\beta(x, y, t), \gamma(x, y, t)$, а первое уравнение используем для нахождения давления:

$$
p(x, y, t)=p(x, y, 0)-\frac{\rho_{0}}{\delta} \int_{0}^{t}\left(u_{x}+v_{y}\right) d t .
$$

Разделим уравнения (2d) и (2f) на $2 \mu$, а уравнение $(2 \mathrm{e})$ - на $\mu$ и запишем систему $(2 \mathrm{~b})-(2 \mathrm{f})$ в матричной форме:

$$
\mathbf{A} \frac{\partial \mathbf{q}}{\partial t}+\mathbf{B} \frac{\partial \mathbf{q}}{\partial x}+\mathbf{C} \frac{\partial \mathbf{q}}{\partial y}+\mathbf{Q q}=0
$$


где $\mathbf{q}=(u, v, \alpha, \beta, \gamma)$,

$$
\begin{aligned}
\mathbf{A} & =\left(\begin{array}{ccccc}
\rho_{0} & 0 & 0 & 0 & 0 \\
0 & \rho_{0} & 0 & 0 & 0 \\
0 & 0 & \frac{\tau}{2 \mu} & 0 & 0 \\
0 & 0 & 0 & \frac{\tau}{\mu} & 0 \\
0 & 0 & 0 & 0 & \frac{\tau}{2 \mu}
\end{array}\right) \\
\mathbf{B} & =\left(\begin{array}{ccccc}
0 & 0 & -1 & 0 & 0 \\
0 & 0 & 0 & -1 & 0 \\
-1 & 0 & 0 & 0 & 0 \\
0 & -1 & 0 & 0 & 0 \\
0 & 0 & 0 & 0 & 0
\end{array}\right) \\
\mathbf{C} & =\left(\begin{array}{ccccc}
0 & 0 & 0 & -1 & 0 \\
0 & 0 & 0 & 0 & -1 \\
0 & 0 & 0 & 0 & 0 \\
-1 & 0 & 0 & 0 & 0 \\
0 & -1 & 0 & 0 & 0
\end{array}\right) \\
\mathbf{Q} & =\left(\begin{array}{ccccc}
0 & 0 & 0 & 0 & 0 \\
0 & 0 & 0 & 0 & 0 \\
0 & 0 & \frac{1}{2 \mu} & 0 & 0 \\
0 & 0 & 0 & \frac{1}{\mu} & 0 \\
0 & 0 & 0 & 0 & \frac{1}{2 \mu}
\end{array}\right) .
\end{aligned}
$$

Система (3) оказывается симметрической $t$-гиперболической (по Фридрихсу) системой, т. к. матрицы $\mathbf{A}, \mathbf{B}, \mathbf{C}$ - симметрические, а матрица $\mathbf{A}$ к тому же положительно определенная в силу положительности констант $\rho_{0}, \tau, \mu$.

3. Единственность задачи Коши. Единственность решения задачи Коши в некоторой области $G$ для симметрической $t$-гиперболической системы доказана [10]. При этом предполагается, что область $G$ ограничена поверхностью $S$, состоящей из двух частей, одна из которых лежит в плоскости $t=0$, а другая является поверхностью $\varphi(x, y, t)=0(\nabla \varphi \neq 0)$. Теорема единственности решения задачи Коши в области $G$ доказана при условии неотрицательности всюду на поверхности $\varphi(x, y, t)=0$ квадратичной формы

$$
\left(\left(n_{1} \mathbf{A}+n_{2} \mathbf{B}+n_{3} \mathbf{C}\right) \mathbf{q}, \mathbf{q}\right),
$$

где $\left(n_{1}, n_{2}, n_{3}\right)$ - вектор нормали к поверхности $\varphi(x, y, t)=0$. Известно, что вектора, отвечающие неотрицательно определенным формам, образуют конус, граница которого называется конусом характеристических нормалей и находится из уравнения

$$
\operatorname{det}\left\|n_{1} \mathbf{A}+n_{2} \mathbf{B}+n_{3} \mathbf{C}\right\|=0 .
$$

Раскрывая определитель, получим:

$$
\begin{aligned}
n_{1} \tau\left(n_{1}^{2} \rho_{0} \tau-n_{2}^{2} \mu\right. & \left.-n_{3}^{2} \mu\right) \times \\
& \times\left(n_{1}^{2} \rho_{0} \tau-2 n_{2}^{2} \mu-2 n_{3}^{2} \mu\right)=0 .
\end{aligned}
$$

Это уравнение определяет плоскость $n_{1}=0$ и два конуса:

$$
n_{1}^{2}=\frac{\mu}{\rho_{0} \tau}\left(n_{2}^{2}+n_{3}^{2}\right) \quad \text { и } \quad n_{1}^{2}=\frac{2 \mu}{\rho_{0} \tau}\left(n_{2}^{2}+n_{3}^{2}\right) .
$$

Они делят все пространство на несколько частей. Доказано [10], что та часть пространства, которая содержит вектор $(1,0,0)$, совпадает с векторами, для которых форма (4) положительно определена. Таким образом, условием неотрицательности квадратичной формы (4) является неравенство:

$$
n_{1} \geq \sqrt{\frac{2 \mu}{\rho_{0} \tau}\left(n_{2}^{2}+n_{3}^{2}\right)} .
$$

Следовательно, если начальные условия заданы при $t=0$, например в круге $x^{2}+y^{2} \leq R^{2}$, то решение задачи Коши однозначно определено внутри конической поверхности

$$
\sqrt{x^{2}+y^{2}}+\sqrt{\frac{2 \mu}{\rho_{0} \tau}} t-R=0
$$

с вершиной в точке $x=0, y=0, t=R \sqrt{\frac{\rho_{0} \tau}{2 \mu}}$. Заметим, что при $t>R \sqrt{\frac{\rho_{0} \tau}{2 \mu}}$ эта поверхность перестает существовать.

4. Смешанная задача. Прежде чем говорить о корректности смешанной задачи для системы (3), приведем ее к каноническому виду, который отличается простотой матриц А и В. Такой канонический вид удобен при изучении задач, в постановках которых выделена ось $x$, например, когда граничные условия ставятся на плоскости $x=0$.

Для этого найдем собственные значения пучка матриц $k \mathbf{A}-\mathbf{B}$, т.е. корни уравнения

$$
\operatorname{det}\|k \mathbf{A}-\mathbf{B}\|=0 .
$$

Получим пять различных собственных чисел:

$$
\begin{aligned}
& k_{1}=\sqrt{\frac{\mu}{\rho_{0} \tau}}, k_{2}=\sqrt{\frac{2 \mu}{\rho_{0} \tau}} \\
& k_{3}=-\sqrt{\frac{\mu}{\rho_{0} \tau}}, k_{4}=-\sqrt{\frac{2 \mu}{\rho_{0} \tau}}, k_{5}=0 .
\end{aligned}
$$

Составим матрицу $\mathbf{Z}$ таким образом, что ее столбцы являются решениями уравнений

$$
\left(k_{j} \mathbf{A}-\mathbf{B}\right)\left(\begin{array}{c}
z_{1 j} \\
\vdots \\
z_{5 j}
\end{array}\right)=\left(\begin{array}{c}
0 \\
\vdots \\
0
\end{array}\right) .
$$


Сделаем в симметрической гиперболической системе (3) подстановку $\mathbf{q}=\mathbf{Z U}$, где $\mathbf{U}=$ $\left(u_{1}, u_{2}, u_{3}, u_{4}, u_{5}\right)$ - новые искомые функции. Затем умножим (3) слева на $\mathbf{Z}^{T}$, получим:

$\mathbf{Z}^{T} \mathbf{A} \mathbf{Z} \frac{\partial \mathbf{U}}{\partial t}+\mathbf{Z}^{T} \mathbf{B Z} \frac{\partial \mathbf{U}}{\partial x}+\mathbf{Z}^{T} \mathbf{C Z} \frac{\partial \mathbf{U}}{\partial y}+\mathbf{Z}^{T} \mathbf{Q Z U}=0$

В силу конструкции $\mathbf{Z}$, получаем: $\mathbf{Z}^{T} \mathbf{A Z}-$ единичная матрица, $\mathbf{Z}^{T} \mathbf{B Z}=\mathbf{K}$ - диагональная матрица, с собственными числами $k_{i}$ по диагонали, $\mathbf{Z}^{T} \mathbf{C Z}=\mathbf{C}_{1}$ и $\mathbf{Z}^{T} \mathbf{Q Z}=\mathbf{Q}_{1}$ - симметричные матрицы. Таким образом, для новых искомых функций

$$
\begin{array}{r}
u_{1}=\sqrt{\frac{\rho_{0}}{2}} v-\sqrt{\frac{\tau}{2 \mu}} \beta, u_{2}=\sqrt{\frac{\rho_{0}}{2}} u-\sqrt{\frac{\tau}{4 \mu}} \alpha, \\
u_{3}=\sqrt{\frac{\rho_{0}}{2}} v+\sqrt{\frac{\tau}{2 \mu}} \beta, u_{4}=\sqrt{\frac{\rho_{0}}{2}} u+\sqrt{\frac{\tau}{4 \mu}} \alpha \\
u_{5}=\sqrt{\frac{\tau}{2 \mu}} \gamma
\end{array}
$$

имеем гиперболическую систему в каноническом виде:

$$
\frac{\partial \mathbf{U}}{\partial t}+\mathbf{K} \frac{\partial \mathbf{U}}{\partial x}+\mathbf{C}_{1} \frac{\partial \mathbf{U}}{\partial y}+\mathbf{Q}_{1} \mathbf{U}=0
$$

Доказано [10], что для симметрической гиперболической системы смешанная задача с диссипативными условиями поставлена корректно. Диссипативными называются такие граничные условия, которые обеспечивают отрицательность квадратичной формы

$$
(\mathbf{K} \mathbf{U}, \mathbf{U})<0 \text {. }
$$

В нашем случае условие (5) означает:

$$
\begin{aligned}
&(\mathbf{K} \mathbf{U}, \mathbf{U})=k_{1} u_{1}^{2}+k_{2} u_{2}^{2}+k_{3} u_{3}^{2}+k_{4} u_{4}^{2}+k_{5} u_{5}^{2}= \\
&=\sqrt{\frac{\mu}{\rho_{0} \tau}}\left(\sqrt{\frac{\rho_{0}}{2}} v-\sqrt{\frac{\tau}{2 \mu}} \beta\right)^{2}+ \\
&+\sqrt{\frac{2 \mu}{\rho_{0} \tau}}\left(\sqrt{\frac{\rho_{0}}{2}} u-\sqrt{\frac{\tau}{4 \mu}} \alpha\right)^{2}- \\
&-\sqrt{\frac{\mu}{\rho_{0} \tau}}\left(\sqrt{\frac{\rho_{0}}{2}} v+\sqrt{\frac{\tau}{2 \mu}} \beta\right)^{2}- \\
&-\sqrt{\frac{2 \mu}{\rho_{0} \tau}}\left(\sqrt{\frac{\rho_{0}}{2}} u+\sqrt{\frac{\tau}{4 \mu}} \alpha\right)^{2}= \\
&-2 v \beta-2 u \alpha<0 .
\end{aligned}
$$

Следовательно, для корректности смешанной задачи для системы (3) условия, задаваемые на границе $x=0$, должны удовлетворять неравенству

$$
\left.(\alpha u+\beta v)\right|_{x=0}>0 .
$$

Заключение. Используя предположения о баротропности и малом изменении давления, удается в линейном приближении расщепить систему, описывающую двумерное движение слабосжимаемой вязкоупругой среды Максвелла на уравнение для определения давления и систему для вектора скоростей и компонент тензора напряжений. Благодаря тому, что полученная система оказывается симметрической $t$-гиперболической системой, найдена область единственности задачи Коши и выписано условие корректности начально краевой задачи для математической модели слабосжимаемой вязкоупругой среды Максвелла.

Автор выражает глубокую благодарность В.В. Пухначеву за плодотворное обсуждение и постоянное внимание к рассматриваемой задаче.

\section{Библиографический список}

1. Астарита Дж., Марручи Дж. Основы гидромеханики неньютоновских жидкостей. - М., 1978.

2. Joseph D.D. Fluid dynamics of viscoelastic fluids. - New York, 1990.

3. Годунов С.К., Роменский Е.И. Элементы механики сплошных сред и законы сохранения. Новосибирск, 1998.

4. Серрин Дж. Математические основы классической механики жидкости. - М., 1963.

5. Пухначев В.В. Точные решения уравнений движения несжимаемой вязкоупругой среды Максвелла. - ПМТФ. - 2009. - Т. 50, № 2 .

6. Ляпидевский В.Ю., Пухначев В.В. Гиперболические подмодели несжимаемой вязкоупру- гой среды Максвелла. - Труды математического института им. В.А. Стеклова. - 2013. - Т. 281.

7. Мещерякова Е.Ю. Групповой анализ уравнений несжимаемой вязкоупругой среды Максвелла. - Изв. Алт. гос. ун-та. 2012. № 1-2 (73).

8. Пухначев В.В. Математическая модель несжимаемой вязкоупругой среды Максвелла. ПМТФ. - 2010. - Т. 51, № 4.

9. Gerritsma M.I., Phillips T.N. On the characteristics and compatibility equations for the UCM model fluid Z.Angew // Math. Mech. 2008. - V. 88, No. 7.

10. Годунов C.K. Уравнения математической физики. - М., 1979. 